\title{
Optimisation en scanographie pédiatrique
}

\author{
C. ETARD ${ }^{1}$, A. BOUETTÉ ${ }^{2}$, J.L. REHEL ${ }^{1}$, B. AUBERT ${ }^{1}$, \\ H. DUCOU LE POINTE ${ }^{2}$ J. DAGOU ${ }^{2}$
}

(Manuscrit reçu le 12 octobre 2009, accepté le 13 novembre 2009)

RÉSUMÉ En 2007, le service de radiologie de l'Hôpital Trousseau (Paris) et l'IRSN ont initié une analyse détaillée des doses délivrées lors d'examens scanographiques chez l'enfant. Cette étude a porté sur trois types d'examen (thorax, abdomen-pelvis et rochers) et trois tranches d'âge (nouveau né à 1 an, 4 à 6 ans et 9 à 11 ans). Une première analyse des doses a été réalisée a posteriori sur l'activité du second semestre 2006. Elle a montré que les protocoles d'acquisition du scanner, qui satisfaisaient aux recommandations dosimétriques en vigueur, étaient mal respectés dans le service : les pratiques étaient hétérogènes et les valeurs moyennes de PDL supérieures aux recommandations. Après ces premiers résultats, une action de sensibilisation à l'optimisation des doses a été menée auprès du personnel et les protocoles d'acquisition du scanner ont été optimisés. L'analyse dosimétrique reprise en 2008 suivant le schéma de 2006 a montré une harmonisation des pratiques au sein du service, une diminution significative des PDL moyens $\mathbf{( - 5 0 \%}$ par exemple en scanographie thoracique) et le respect systématique des recommandations dosimétriques pour les trois examens étudiés.

ABSTRACT Optimization in paediatric computed tomography.

The Department of radiology of the Hospital Trousseau (Paris) and IRSN have initiated in 2007 a detailed analysis of doses delivered to children undergoing CT examinations. This study involved three types of CT examination (chest, abdomen + pelvis and petrosal bones) and three age groups (new born to 1 year, 4 to 6 years and 9 to 11 years). A first analysis of doses was realised on a six months period of activity at the end of 2006. It stated that the standardized protocols of the scanner, that satisfied the relevant dosimetric requirements, were regularly modified by the operator to acquire the CT slides: the practice among the department was heterogeneous and the mean values of DLP were systematically higher than those recommended. The whole staff of the department has been informed of these first results and made aware of optimization of delivered doses. The standardized protocols of the scanner have been optimized. A second analysis, similar to the first one, was conducted in 2008 . It showed the harmonization of the practice among the department, a significant decrease in the mean DLP $(-50 \%$ for chest CT) and the respect for the dosimetric requirements for the three examinations concerned.

Keywords: Medical exposure / optimization / dose length product / computed tomography

\section{Introduction}

Depuis la transposition de la directive européenne Euratom 97/43 (1997), la justification et l'optimisation des pratiques jouent un rôle prépondérant dans la radioprotection des patients.

\footnotetext{
Institut de radioprotection et de sûreté nucléaire, DRPH/SER/UEM, BP 17, 92262 Fontenay-aux-Roses, France.

Hôpital Armand-Trousseau, 26 avenue du Dr. Arnold-Netter, 75012 Paris, France.
} 
Ces deux principes concernent tous les patients mais ils doivent s'appliquer avec encore plus de rigueur chez les enfants. En effet, ces derniers sont plus sensibles aux rayonnements ionisants que les adultes.

À l'hôpital Armand Trousseau (AP-HP ${ }^{3}$ ), qui a pour principale vocation la prise en charge des enfants et des adolescents, le service de radiologie s'est engagé depuis plusieurs années dans la mise en application de ces principes de radioprotection. Ainsi, en scanographie, des protocoles ont été établis en relation avec le constructeur, selon l'âge et/ou le poids des patients, pour respecter les recommandations dosimétriques de la Société francophone d'imagerie pédiatrique et prénatale (SFIPP).

Bien que les pratiques du service soient basées sur des protocoles satisfaisants, le service et l'Institut de radioprotection et de sûreté nucléaire (IRSN) ont initié début 2007 une analyse des doses réellement délivrées. Cette première analyse ayant mis en évidence des dérives dans les pratiques conduisant à des doses parfois très supérieures aux valeurs recommandées, des actions correctrices ont été menées et l'analyse des doses a été renouvelée en 2008.

Cet article présente les principaux résultats obtenus en 2007 et l'apport des actions d'optimisation sur les doses délivrées aux patients.

\section{Matériel et méthodes}

\subsection{Examens retenus pour l'étude}

L'étude a été réalisée a posteriori et a porté sur trois types d'examens scanographiques fréquemment réalisés dans le service: scanner standard du thorax, scanner abdomino-pelvien et scanner des rochers. Les doses délivrées ont été analysées pour trois tranches d'âge : nouveau né à 1 an (2 ans pour l'examen des rochers), 4 à 6 ans et 9 à 11 ans.

Des périodes d'activité d'environ 6 mois ont été analysées. L'activité de septembre 2006 à février 2007 a été étudiée en juillet 2007 et une seconde analyse portant sur l'activité de mars à septembre 2008 a été réalisée en décembre 2008.

Les protocoles d'acquisition définis sur le scanner du service ${ }^{4}$ pour ces types d'examens et ces tranches d'âge sont présentés dans le tableau I.

Assistance Publique-Hôpitaux de Paris.

4 Scanner Philips brillance 16 coupes. 
OPTIMISATION EN SCANOGRAPHIE PÉDIATRIQUE

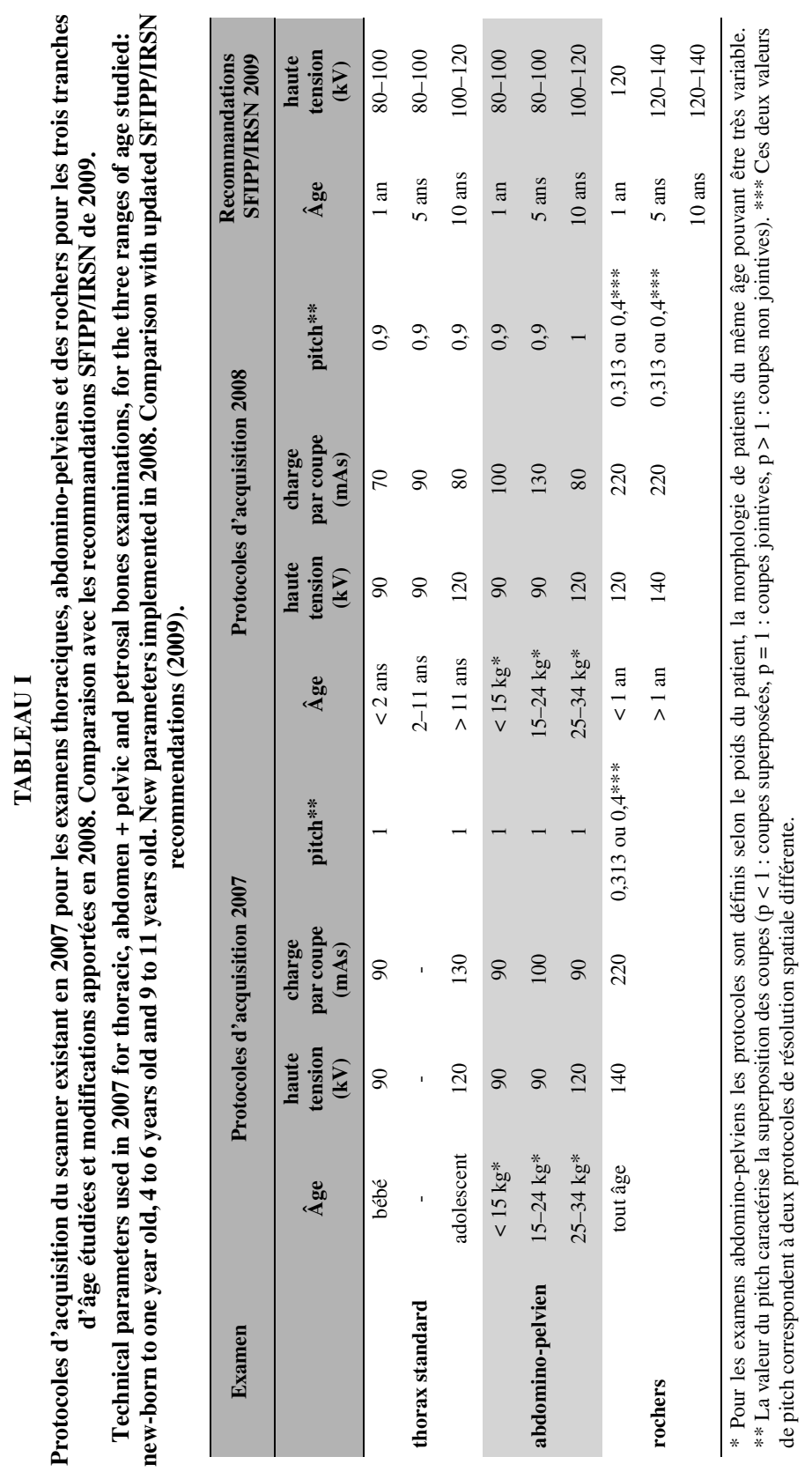




\subsection{Origines des données}

Grâce au logiciel de gestion administrative de l'activité du service ${ }^{5}$, les informations nécessaires à l'étude ont pu être obtenues pour chaque examen scanographique :

- le type d'examen, selon la classification commune de actes médicaux,

- l'âge du patient,

- le produit dose $\times$ longueur (PDL) de l'examen complet, éventuellement constitué de plusieurs acquisitions, seul paramètre dosimétrique enregistré systématiquement.

Le PDL est une grandeur dosimétrique spécifique à la scanographie. Il est égal au produit de l'indice de dose scanographique volumique ${ }^{6}$ par la longueur explorée. Il caractérise ainsi la dose délivrée au volume étudié.

Afin de permettre des comparaisons avec des valeurs de référence, le PDL par acquisition a été déterminé pour chaque examen. Le nombre d'acquisitions réalisées pour chaque examen a été obtenu en consultant le système d'archivage d'images du service. Tous les résultats de l'étude sont donc exprimés en termes de PDL par acquisition (en mGy.cm).

\subsection{Références dosimétriques en scanographie pédiatrique}

Il n'existe pas à ce jour de niveaux de référence diagnostiques en scanographie pédiatrique. Les recommandations dosimétriques de la SFIPP publiées dans les guides de procédures radiologiques (SFR, 2006), ont donc été prises pour référence pour évaluer les pratiques du service en termes dosimétriques.

\section{Résultats et analyse}

\section{1. État des lieux de fin 2006 à début 2007}

Une première analyse des valeurs de PDL a été réalisée en juillet 2007 sur l'activité de septembre 2006 à février 2007.

\subsubsection{Examen du thorax standard}

Les résultats obtenus pour l'examen thoracique sont présentés sur la figure 1 .

\footnotetext{
5 Logiciel « Rados », mis en place au niveau de l'AP-HP.

6 Ce paramètre étant indépendant du nombre d'acquisitions réalisés et de la longueur explorée, il peut être intéressant, lorsqu'il est disponible, de l'utiliser également comme paramètre de référence pour une étude d'optimisation. 


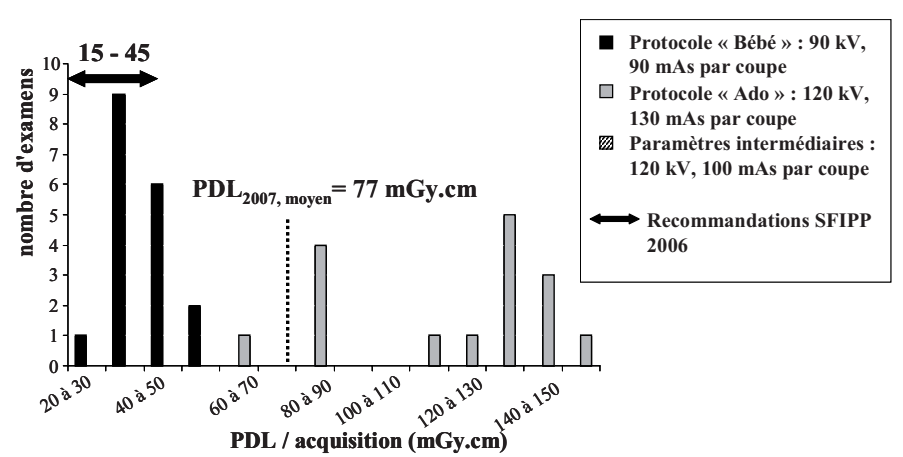

(a)

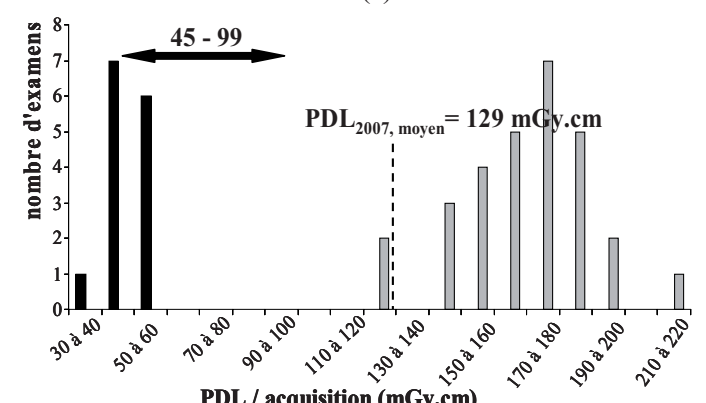

(b)

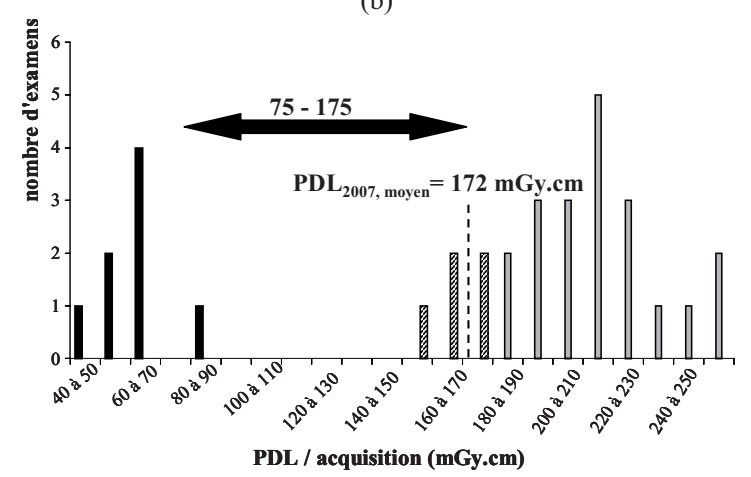

(c)

Figure 1 - Distribution des PDL par acquisition pour les examens du thorax réalisés de septembre 2006 à février 2007. Caractéristiques des protocoles utilisés et recommandations dosimétriques de la SFIPP en vigueur (2006) ; (a) nouveau-né à 1 an, (b) enfant de 4 à 6 ans, (c) enfant de 9 à 11 ans.

Distribution of the DLP by scan, for thoracic examinations realized from September 2006 to February 2007. Technical parameters used and current recommendations of the SFIPP (2006); (a) new-born to 1 year old, (b) child from 4 to 6 years old, (c) child from 9 to 11 years old. 
Pour les trois tranches d'âge étudiées, les valeurs moyennes de PDL par acquisition sont dans la fourchette haute, voire supérieures aux recommandations de la SFIPP. De plus, la grande dispersion des valeurs de PDL par acquisition pour une même tranche d'âge semble systématiquement indiquer deux pratiques distinctes au sein du service.

Pour expliquer ces résultats, les paramètres d'acquisition réellement utilisés (haute tension et charge électrique par rotation) ont été relevés pour chaque examen dans le système d'archivage d'image afin de vérifier s'ils correspondaient aux deux protocoles « bébé » et « adolescent » définis par le service à cette époque (Tab. I).

Les résultats présentés sur la figure 1 montrent que près de $50 \%$ des examens des bébés (nouveau né à 1 an) étaient réalisés avec les paramètres du protocole «adolescent» et conduisaient à des PDL jusqu'à 4 fois supérieurs aux recommandations de la SFIPP (Fig. 1a). Ces recommandations étaient par contre respectées pour les examens réalisés avec les paramètres du protocole « bébé ».

L'inexistence de protocole défini pour les enfants d'âge intermédiaire (entre bébés et adolescents) a conduit, pour $67 \%$ des enfants de 4 à 6 ans et $86 \%$ des enfants de 9 à 11 ans, à l'utilisation du protocole « adolescent » ou de paramètres intermédiaires.

Ces pratiques hétérogènes expliquent, pour l'examen thoracique, la forme des distributions présentées sur la figure 1.

\subsubsection{Examen abdomino-pelvien}

Pour ce type d'examen, le nombre d'examens par tranche d'âge dans la période considérée est faible, les examens incluant le thorax, très fréquents, ne pouvant être inclus dans l'étude. Néanmoins, l'analyse a pu mettre en évidence que bien qu'il existait des protocoles définis par tranche de poids de l'enfant, les examens étaient parfois réalisés avec des paramètres correspondant à un poids supérieur à celui du patient et conduisant à des PDL supérieurs aux recommandations de la SFIPP.

\subsubsection{Examen des rochers}

Les résultats obtenus pour l'examen des rochers sont présentés sur la figure 2.

Pour les trois tranches d'âge étudiées, les valeurs de PDL par acquisition sont moins dispersées que celles constatées pour les examens thoraciques car la longueur explorée fluctue peu d'un enfant à l'autre (de 3 à $4 \mathrm{~cm}$ explorés selon la tranche d'âge) et est relativement indépendante de la morphologie de l'enfant au sein d'une même tranche d'âge. 


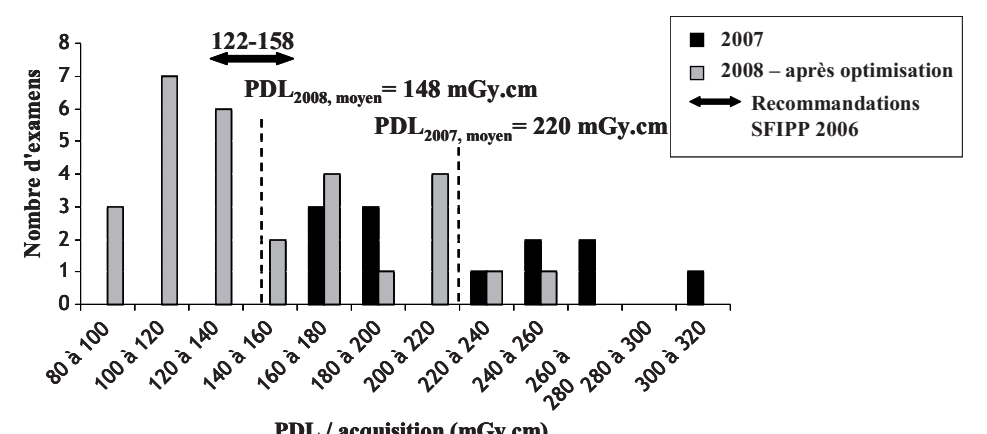

PDL / acquisition (mGy.cm)

(a)

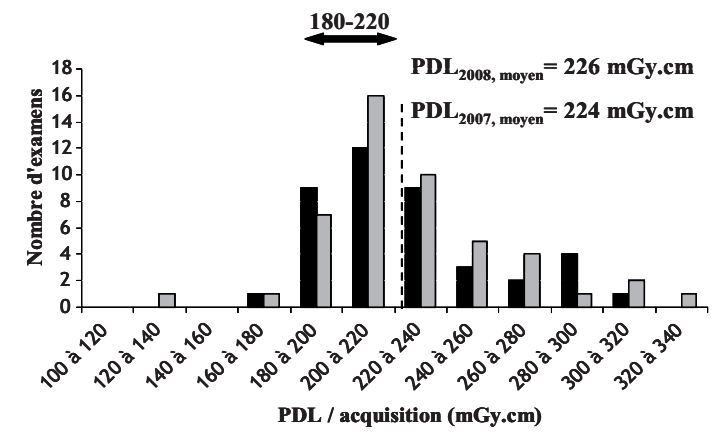

(b)

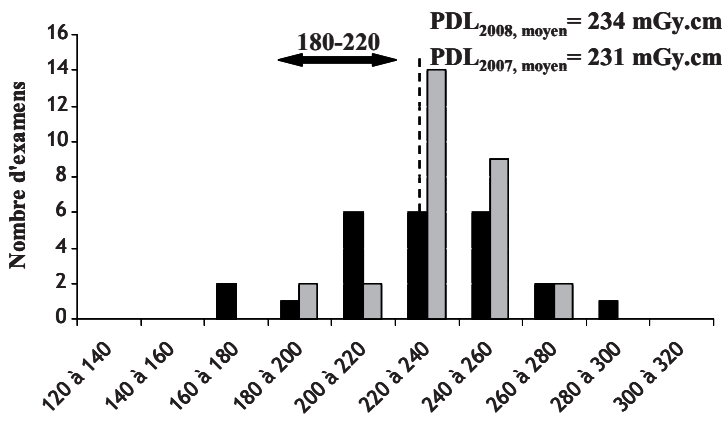

PDL / acquisition (mGy.cm)

(c)

Figure 2 - Distribution des PDL par acquisition pour les examens des rochers réalisés de septembre 2006 à février 2007 et après optimisation, de mars à septembre 2008. Recommandations de la SFIPP en vigueur (2006); (a) nouveau-né à 2 ans, (b) enfant de 4 à 6 ans, (c) enfant de 9 à 11 ans. Distribution of the DLP by scan, for examinations of petrosal bones realized from September 2006 to February 2007 and after optimization from March to September 2008. Current recommendations of the SFIPP (2006); (a) new-born to 1 year old, (b) child from 4 to 6 years old, (c) child from 9 to 11 years old. 
Les valeurs moyennes de PDL par acquisition sont très légèrement supérieures aux recommandations de la SFIPP pour les enfants de 4 à 6 ans et de 9 à 11 ans. Pour la tranche d'âge du nouveau-né à 2 ans, le PDL moyen par acquisition est très supérieur à la valeur recommandée. Cet écart s'explique en partie par le choix du service de limiter les sédations chez les tout-petits. Les mouvements du patient rendent alors plus délicat le centrage sur la zone à explorer et conduisent l'opérateur à prendre une marge de sécurité en augmentant la longueur explorée.

\subsection{Actions correctrices entreprises au sein du service}

Le personnel du service, radiologues et manipulateurs, a été informé des résultats de cette première analyse. Afin de corriger les dérives décelées dans les pratiques, ils ont été à nouveau sensibilisés à la radioprotection de leurs jeunes patients, aux paramètres qui influent sur les doses délivrées et à la nécessité de respecter les protocoles établis.

Le problème de la qualité des images a été abordé avec les radiologues afin qu'ils prennent conscience qu'une image très, ou trop belle est une image plus irradiante pour le patient, sans apport diagnostic (Siegel et al., 2004). La longueur de la zone explorée est également à optimiser.

Enfin, une action a été menée fin 2007 avec le constructeur du scanner pour modifier certains protocoles d'acquisition et ajouter des protocoles pour les tranches d'âge intermédiaires, qui n'existaient pas en particulier pour l'examen thoracique.

Les nouveaux protocoles d'acquisition sont présentés dans le tableau I. Ils ont été validés par les cliniciens quant à la qualité diagnostique des images et, par ailleurs, ils répondent aux recommandations les plus récentes de la SFIPP et de l'IRSN (Brisse et Aubert, 2009).

\subsection{Apport des actions d'optimisation}

L'étude a été réitérée en décembre 2008 sur l'activité de mars à septembre 2008, pour les mêmes examens et les mêmes tranches d'âge.

\subsubsection{Examen du thorax standard}

Les résultats obtenus pour l'examen du thorax sont présentés sur la figure 3. Leur analyse montre, pour les trois tranches d'âge étudiées :

- une forte diminution des valeurs moyennes de PDL par acquisition (environ $50 \%)$; 


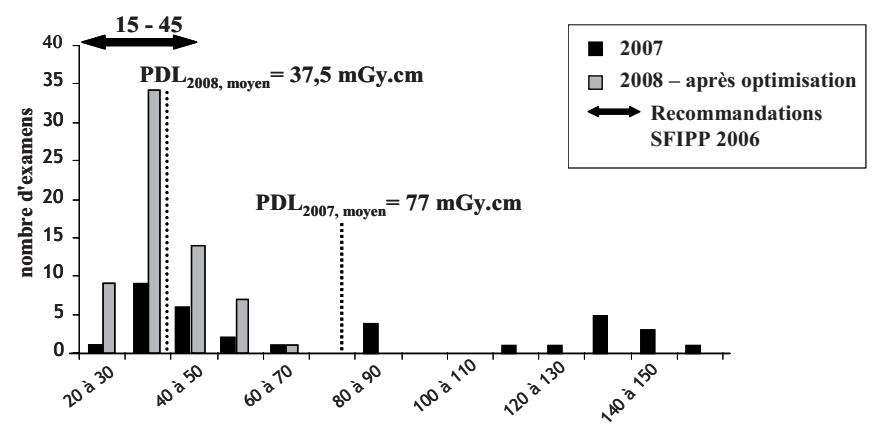

PDL / acquisition (mGy.cm)

(a)

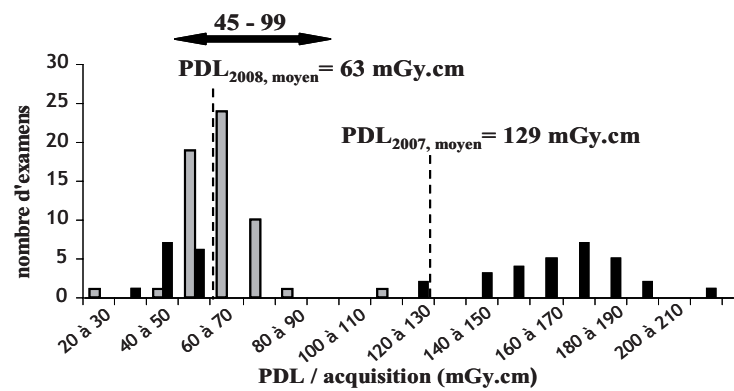

(b)

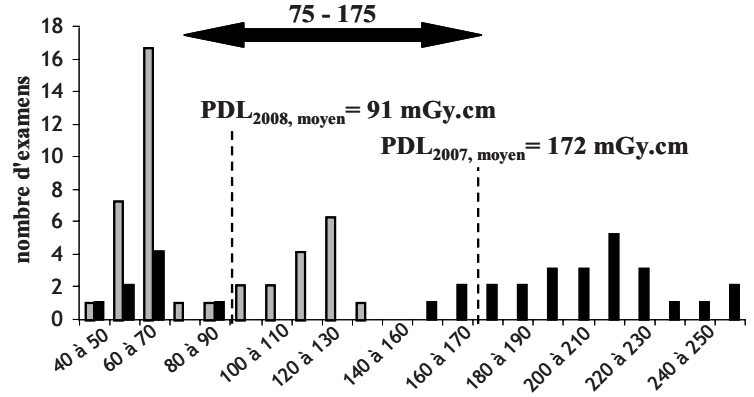

PDL / acquisition (mGy.cm)

(c)

Figure 3 - Distribution des PDL par acquisition pour les examens du thorax réalisés de septembre 2006 à février 2007, et après optimisation de mars à septembre 2008. Recommandations de la SFIPP en vigueur (2006); (a) nouveau-né à 1 an, (b) enfant de 4 à 6 ans, (c) enfant de 9 à 11 ans.

Distribution of the DLP by scan, for thoracic examinations realized from September 2006 to February 2007 and after optimization from March to September 2008. Current recommendations of the SFIPP (2006); (a) new-born to 1 year old, (b) child from 4 to 6 years old, (c) child from 9 to 11 years old. 
- le respect des recommandations de la SFIPP ;

- une distribution des PDL beaucoup plus resserrée autour de la valeur moyenne, qui montre une harmonisation des pratiques dans le service.

\subsubsection{Examen abdomino-pelvien}

Les résultats obtenus pour l'examen du thorax sont présentés sur la figure 4. Leur analyse montre, pour toutes les tranches d'âge :

- le respect des recommandations de la SFIPP;

- une distribution des PDL resserrée autour de la valeur moyenne, qui montre une homogénéité dans les pratiques au sein du service.

Même si le faible nombre de données disponibles sur la première période d'analyse (2006/2007) ne permet pas une comparaison rigoureuse des pratiques entre les deux phases de l'étude, il semble que celles-ci se soient harmonisées.

\subsubsection{Examen des rochers}

Les résultats obtenus pour l'examen des rochers sont présentés sur la figure 2. Leur analyse montre, pour les trois tranches d'âge étudiées :

- une nette diminution des valeurs de PDL par acquisition pour la tranche d'âge du nouveau-né à deux ans (diminution de $33 \%$ de la valeur moyenne);

- le respect des recommandations de la SFIPP pour la tranche d'âge du nouveauné à 2 ans ;

- la stabilité des PDL par acquisition relevés pour les tranches d'âge de 4 à 6 ans et de 9 à 11 ans.

Il convient par d'ailleurs de noter que, suite à une enquête réalisée en 2008, la SFIPP et l'IRSN ont récemment publié des valeurs de référence plus élevées (Brisse et Aubert, 2009), respectivement égales à 157, 280 et $340 \mathrm{mGy} . \mathrm{cm}$ pour les trois tranches d'âge de cette étude. Les valeurs relevées en 2008 dans le service respectent ces nouvelles recommandations.

\section{Conclusion}

Cette étude a montré que la recherche de l'image « la plus belle » peut induire des dérives dans les pratiques, que seule une analyse détaillée permet de déceler. Celle-ci a permis de constater que, bien que des protocoles d'acquisition aient été définis sur le scanner en accord avec les recommandations de la SFIPP, ceux-ci n'étaient pas systématiquement respectés par l'ensemble des radiologues et des manipulateurs du service. 


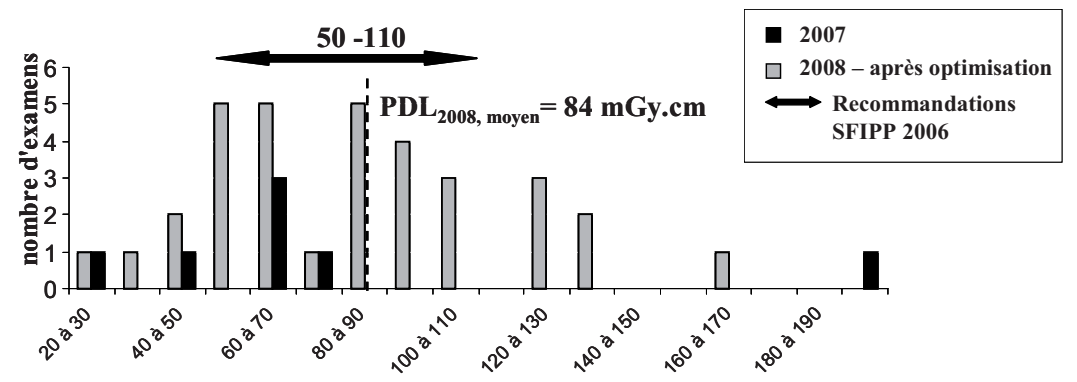

PDL / acquisition (mGy.cm)

(a)

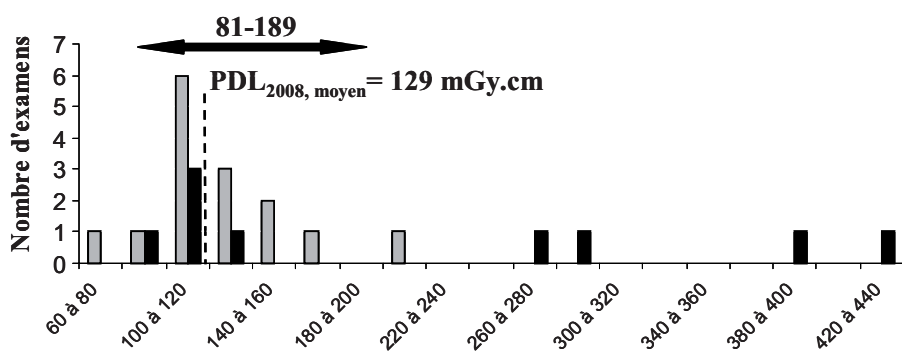

PDL / acquisition (mGy.cm)

(b)

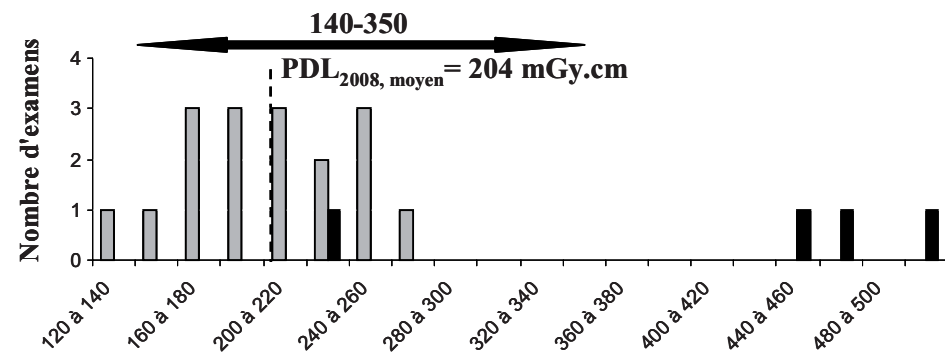

PDL / acquisition (mGy.cm)

(c)

Figure 4-Distribution des PDL par acquisition pour les examens abdomino-pelviens réalisés de septembre 2006 à février 2007, et après optimisation, de mars à septembre 2008. Recommandations de la SFIPP en vigueur (2006); (a) nouveau-né à 1 an, (b) enfant de 4 à 6 ans, (c) enfant de 9 à 11 ans.

Distribution of the DLP by scan, for abdomen and pelvic examinations realized from September 2006 to February 2007 and after optimization from March to September 2008. Current recommendations of the SFIPP (2006); (a) new-born to 1 year old, (b) child from 4 to 6 years old, (c) child from 9 to 11 years old. 
Les protocoles proposés par les constructeurs sont rarement optimisés. Une action volontariste des services est nécessaire, mais possible, auprès des constructeurs qui peuvent modifier les paramètres des protocoles pour diminuer les doses tout en gardant une qualité d'image suffisante pour les cliniciens. Il est à noter que ce type d'étude entre dans les missions de la personne spécialisée en radiophysique médicale.

En combinant formation du personnel et action auprès du constructeur, les résultats obtenus montrent qu'il est possible de réduire de façon significative les doses délivrées au patient en scanographie pédiatrique, tout en gardant une qualité d'image tout à fait compatible avec le diagnostic.

Enfin, cette étude montre que la mise en application du principe d'optimisation est compatible avec la pratique clinique au quotidien. Elle nécessite l'implication et l'adhésion de tout le personnel, radiologues et manipulateurs, qui doivent accepter de modifier leurs façons de travailler et rester vigilants afin que les résultats très encourageants obtenus ne dérivent pas au fil du temps.

\section{RÉFÉRENCES}

Brisse H., Aubert B. (2009) Niveaux d'exposition en tomodensitométrie multicoupes pédiatrique : résultats de l'enquête dosimétrique SFIPP/IRSN 2007-2008, J. Radiol. 90, 207-215.

Euratom (1997) Directive 97/43 Euratom du 30 juin 1997, relative à la protection des personnes contre les dangers des rayonnements ionisants lors d'exposition à des fins médicales ; disponible sur http://eur-lex.europa.eu.

SFR (2006) Guide de procédures radiologiques SFR/IRSN, Scanographie pédiatrique, mise à jour 01/2006 ; disponible sur www.sfip-radiopediatrie.org.

Siegel M.J., Schmidt B., Bradley D., et al. (2004) Radiation dose and image quality in pediatric CT: effect of technical factors and phantom size and shape, Radiology 233, 515-522. 